\title{
Subarcsecond bright points and quasi-periodic upflows below a quiescent filament observed by IRIS
}

\author{
T. Li and J. Zhang
}

\begin{abstract}
Key Laboratory of Solar Activity, National Astronomical Observatories, Chinese Academy of Sciences, 100012 Beijing, PR China e-mail: liting@nao.cas.cn
\end{abstract}

Received 30 July 2015 / Accepted 8 March 2016

\begin{abstract}
Context. The new Interface Region Imaging Spectrograph (IRIS) mission provides high-resolution observations of UV spectra and slit-jaw images (SJIs). These data have become available for investigating the dynamic features in the transition region (TR) below the on-disk filaments.

Aims. The driver of "counter-streaming" flows along the filament spine is still unknown yet. The magnetic structures and the upflows at the footpoints of the filaments and their relations with the filament mainbody have not been well understood. We study the dynamic evolution at the footpoints of filaments in order to find some clues for solving these questions.

Methods. Using UV spectra and SJIs from the IRIS, along with coronal images and magnetograms from the Solar Dynamics Observatory (SDO), we present the new features in a quiescent filament channel: subarcsecond bright points (BPs) and quasi-periodic upflows.

Results. The BPs in the TR have a spatial scale of about 350-580 km and lifetimes of more than several tens of minutes. They are located at stronger magnetic structures in the filament channel with a magnetic flux of about $10^{17}-10^{18} \mathrm{Mx}$. Quasi-periodic brightenings and upflows are observed in the BPs, and the period is about 4-5 min. The BP and the associated jet-like upflow comprise a "tadpole-shaped" structure. The upflows move along bright filament threads, and their directions are almost parallel to the spine of the filament. The upflows initiated from the BPs with opposite polarity magnetic fields have opposite directions. The velocity of the upflows in the plane of sky is about $5-50 \mathrm{~km} \mathrm{~s}^{-1}$. The emission line of Si IV $1402.77 \AA$ at the locations of upflows exhibits obvious blueshifts of about $5-30 \mathrm{~km} \mathrm{~s}^{-1}$, and the line profile is broadened with the width of more than $20 \mathrm{~km} \mathrm{~s}^{-1}$.

Conclusions. The BPs seem to be the bases of filament threads, and the upflows are able to convey mass for the dynamic balance of the filament. The "counter-streaming" flows in previous observations may be caused by the propagation of bi-directional upflows initiated from opposite polarity magnetic fields. We suggest that quasi-periodic brightenings of BPs and quasi-periodic upflows result from small-scale oscillatory magnetic reconnections, which are modulated by solar p-mode waves.
\end{abstract}

Key words. Sun: filaments, prominences - Sun: transition region - Sun: UV radiation

\section{Introduction}

Quiescent filaments are large structures of cool and dense plasma embedded in the surrounding hotter corona of quiet regions. Despite over a century of observations, a big question still remains: how do the filament plasma form and evolve. It is recognized that the filament material should come from the chromosphere, rather than the rarefied corona (Pikel'Ner 1971; Saito \& Tandberg-Hanssen 1973). Several models have been proposed to explain the formation process of filament material. In injection models, chromospheric plasma is propelled directly into the corona through the reconnection of magnetic field during canceling flux (Wang 1999; Chae et al. 2001; Litvinenko et al. 2007). Levitation models propose that cool plasma is lifted by flux rope emergence from below the photosphere or the post-reconnection relaxation associated with flux cancellation (van Ballegooijen \& Martens 1989; Priest et al. 1996). In evaporation-condensation models, chromospheric plasma is evaporated owing to thermal non-equilibrium and ultimately condensed in the corona to form cool filament threads (Karpen et al. 2005; Xia et al. 2012; Liu et al. 2012; Berger et al. 2012). The dynamic evolution at the footpoints of the filaments could provide important information for our understanding of the formation mechanism of the filaments (Parenti 2014; Li et al. 2015).

Bright points (BPs) are ubiquitous small-scale bright dynamical features at various atmosphere layers. BPs are frequently observed in X-rays and extreme ultraviolet (EUV) wavelengths and correspond to coronal bright points (CBPs). The spatial sizes of CBPs are around 5-20" and their lifetime ranges from several hours to two days (Vaiana et al. 1973; Golub et al. 1974; Li et al. 2013). CBPs are often located at network magnetic features of opposite polarity with the total fluxes of about $10^{19}-10^{20} \mathrm{Mx}$ (Golub et al. 1976; Hong et al. 2014). The observations showed that some CBPs are associated with quasi-periodic impulsive flashes with periods of a few minutes to hours (Habbal et al. 1990; Ning \& Guo 2014; Samanta et al. 2015). Some authors suggest that small-scale repeated magnetic reconnections result in the quasi-periodic variation in the intensity of CBPs (Madjarska et al. 2003; Doyle et al. 2006; Zhang et al. 2012). Recently, Régnier et al. (2014) reported the existence of subarcsecond EUV bright dots at the edge of the active region with a characteristic length of $680 \mathrm{~km}$ from observations of the Highresolution Coronal (Hi-C) instrument. The bright dots are shortlived (25 s) and appear at the footpoints of large-scale coronal 
loops. Tian et al. (2014a) found similar subarcsecond bright dots above sunspots in the transition region (TR) with the observations of the Interface Region Imaging Spectrograph (IRIS; De Pontieu et al. 2014).

The majority of previous observations about filaments were taken in $\mathrm{H} \alpha$ and EUV wavelengths, and the dynamical features below the on-disk filaments could not be seen as a result of the abundant Lyman continuum absorption and the "volumeblocking" effect (Wang et al. 1998; Heinzel et al. 2001). The recently launched IRIS mission is now providing high-resolution observations of the TR and the chromosphere. The slit-jaw images (SJIs) from the IRIS are centered at 1330, 1400, 2796, and $2832 \AA$ (De Pontieu et al. 2014). The filaments seem semitransparent and optically thin in the channels of 1330 and $1400 \AA$, and the dynamic evolution at the footpoints of the filaments could be seen. Thus in this work we investigate the small-scale activities at the footpoints of the filament in the TR by combining imaging and spectroscopic observations of the IRIS and try to find some clues to the material source of the filaments. Here, new dynamical features in the TR below the quiescent filaments are found: subarcsecond BPs and quasiperiodic upflows. We investigate the physical properties of BPs, the quasi-periodic patterns of BPs, and associated upflows.

\section{Observations and data analysis}

The IRIS observations were taken from 18:01 UT to 18:45 UT on 2015 February 13, with the field of view (FOV) covering part of a quiescent filament in the southern hemisphere. The SJIs centered at 1330,1400 , and $2796 \AA$ were used to analyze the evolution of BPs with 0 '.33-0 '.4 spatial resolution. The observations had a long exposure time of $15 \mathrm{~s}$ and a temporal cadence of $\sim 66 \mathrm{~s}$. The spectral data are taken in an eight-step raster mode with a step cadence of $16.4 \mathrm{~s}$ and a spectral dispersion of $\sim 0.025 \AA$ pixel $^{-1}$. The SJIs at $1330 \AA$ and spectral data of the Si IV $1402.77 \AA$ line were mainly analyzed. The C II $1335.71 \AA$ line is formed in the lower TR and corresponds to a temperature of about $2.5 \times 10^{4} \mathrm{~K}$ ( $\mathrm{Li}$ et al. 2014). The Si IV $1402.77 \AA$ line is formed in the middle TR with a temperature of about $8.0 \times 10^{4} \mathrm{~K}$. The calibrated level-2 data were used in our study. The EUV observations from the Atmospheric Imaging Assembly (AIA; Lemen et al. 2012) onboard the Solar Dynamics Observatory (SDO; Pesnell et al. 2012) were used to compare with the IRIS data. Cross-correlation between the AIA $1600 \AA$ image and the IRIS SJI $1400 \AA$ image was used for the coalignment (Tian et al. 2014b). The full-disk line-ofsight magnetograms from the Helioseismic and Magnetic Imager (HMI; Scherrer et al. 2012) were also applied to analyze the magnetic structures at the BPs.

\section{Results}

The large-scale quiescent filament stretches across the solar southern hemisphere with a length of $\sim 1000 \mathrm{Mm}$ (Fig. 1a). The middle part of the filament is covered by the FOV of the IRIS $\left(148^{\prime \prime} \times 148^{\prime \prime}\right)$. In the zoomed $171 \AA$ image (Fig. 1b), several bright thread-like structures were observed along the spine of the filament. At the bases of these threads, a few grain-like knots could be obscurely seen. High-resolution observations of 1330 and $1400 \AA$ clearly showed several tens of small-scale BPs underlying the filament (Figs. 1c-d). These BPs are mainly located in two areas below the filament (regions "R1" and "R2" in
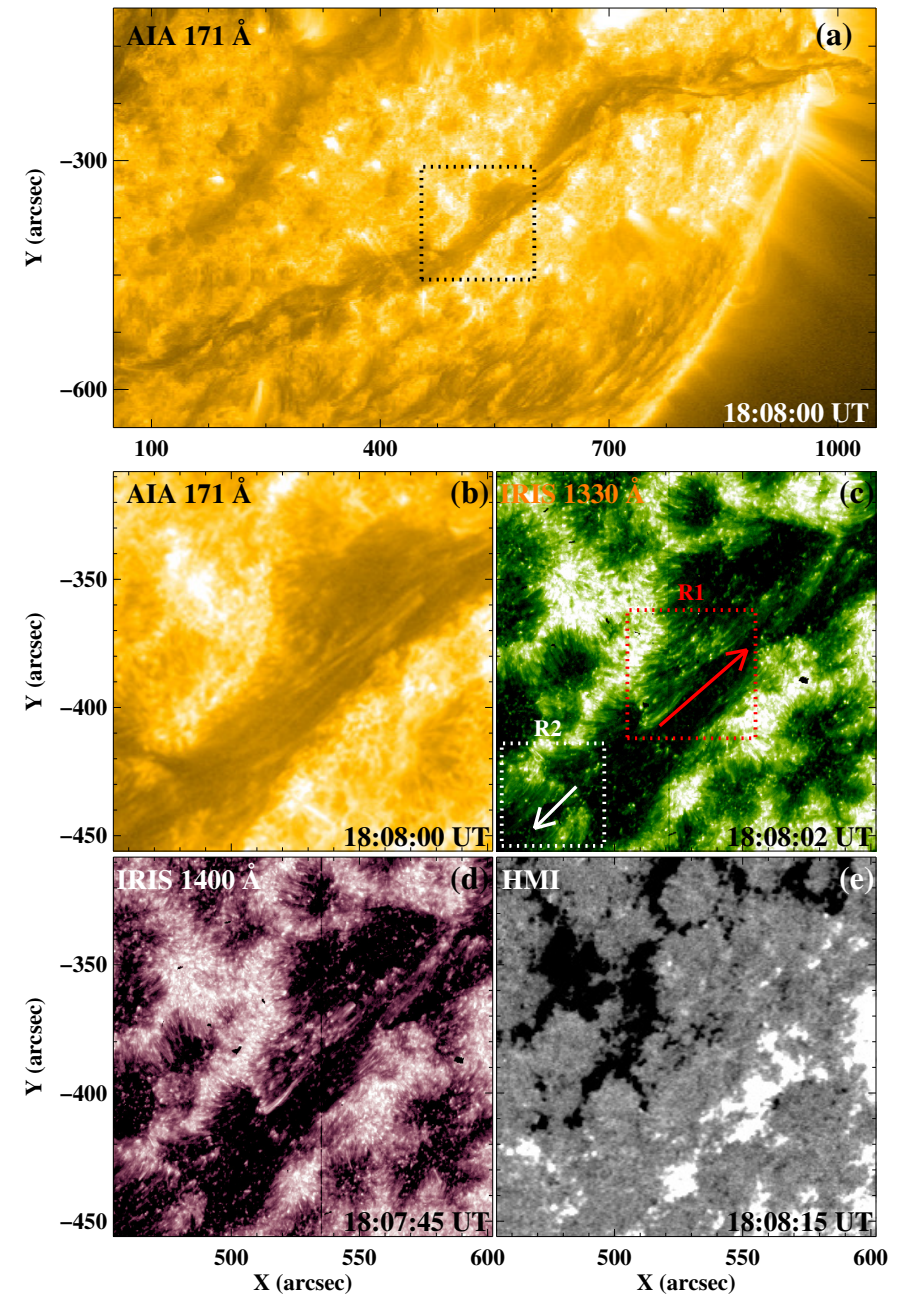

Fig. 1. Panel a): SDO/AIA $171 \AA$ image showing the large quiescent filament on $2015 \mathrm{Feb}$ 13. The black square displays the FOV of the zoomed images in panels b)-e). Panels b)-e): multiwavelength images of SDO/AIA, IRIS $1330 \AA$, IRIS $1400 \AA$, and SDO/HMI LOS magnetogram showing the subarcsecond BPs underlying the filament and bidirectional (red and white arrows) loop-like structures at regions "R1" (red square) and "R2" (white square).

Fig. 1c). Most of the BPs in regions "R1" and "R2" are located at opposite polarity magnetic fields (Fig. 1e). We selected 22 relatively isolated BPs in the two regions and obtained the intensitylocation profiles along the slices that pass through the centers of the BPs. Then the Gaussian function was used to fit the profiles and the full width at half maximum (FWHW) of the Gaussian fitting profile is thought to be the spatial size of each BP. The average size of these BPs is $440 \mathrm{~km}$ (about 4 pixels) with the maximum value of $580 \mathrm{~km}$ and the minimum value of $350 \mathrm{~km}$. Most of the BPs always exist during the observational time interval, and this means that their lifetimes are at least tens of minutes.

The BPs seem to be connected with loop-like structures, appearing to have the morphology of "tadpoles". In region "R1", the directions of the loop-like structures are roughly parallel to the spine of the filament, pointing from the BPs to the northwest (Figs. 2b and e). The comparison of the IRIS and AIA images suggests that several loop-like structures at 1330 and $1400 \AA$ could be observed at 171 and $304 \AA$, corresponding to the bright EUV threads of the filament (arrows in Figs. 2a, b and d, e). The BPs are located at the negative-polarity fields (Fig. 2c) near the polarity inversion line (PIL). Several relatively isolated 

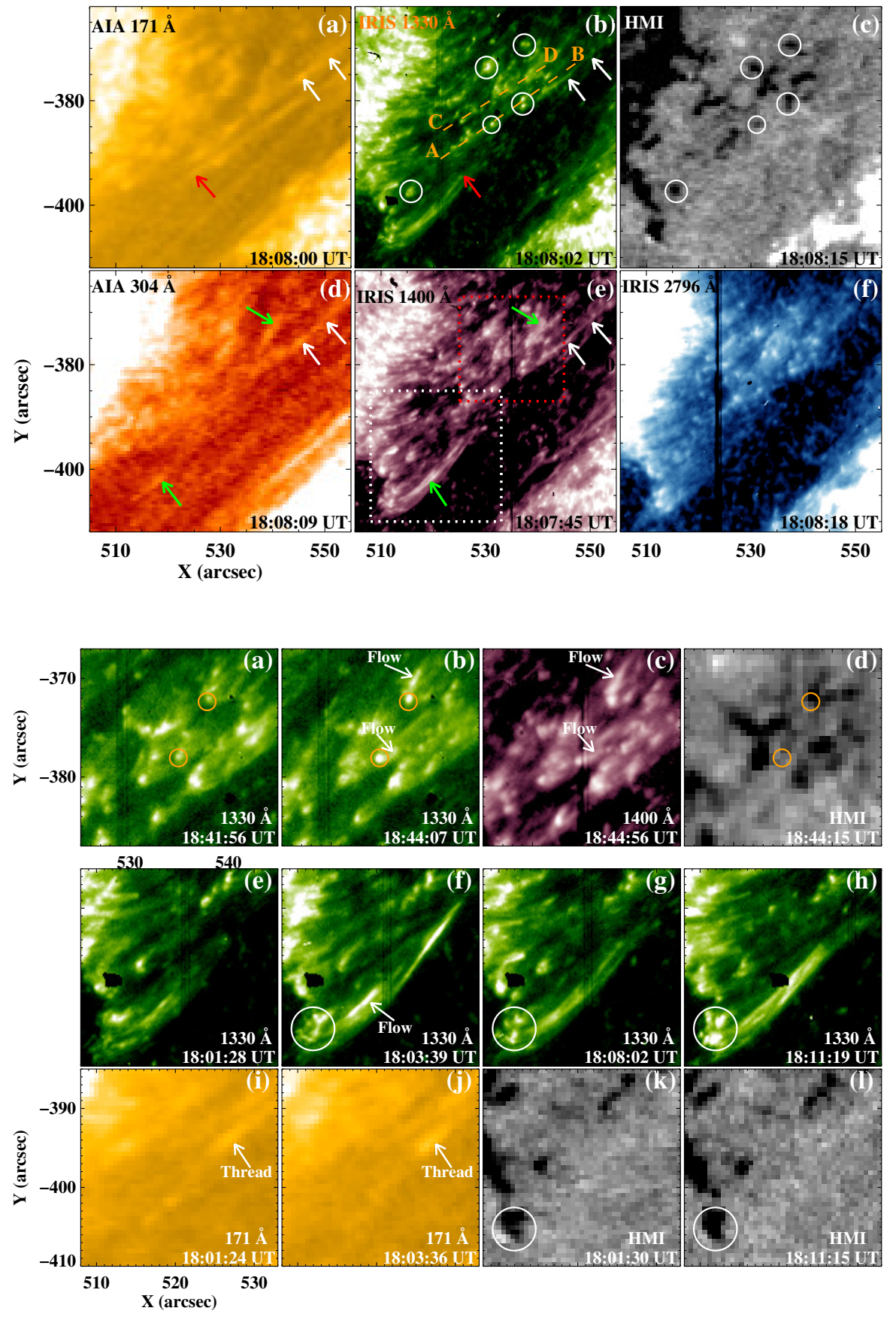

1330
Fig. 2. Appearance of BPs and loop-like structures in region "R1". The white circles outline the locations of several BPs in the $1330 \AA$ image and HMI LOS magnetogram. The arrows in panels a), b) and d), e) point to the looplike structures in close proximity to some BPs. Dashed lines "A-B" and "C-D" (panel b)) show the cut positions used to obtain the stack plots shown in Figs. 5a, b. Red and white squares in panel e) denote the FOVs of Figs. 3a-d and e-1, respectively.
BPs are defined, and their magnetic flux (see white circles in Figs. 2b, c) was calculated. The magnetic flux of these BPs is between $3.8 \times 10^{17} \mathrm{Mx}$ and $1.2 \times 10^{18} \mathrm{Mx}$, and the average value is about $7.0 \times 10^{17} \mathrm{Mx}$. Signatures of these BPs and loop-like structures are not very prominent in the chromospheric $2796 \AA$ images (Fig. 2f).

The temporal evolution of some BPs at region " $\mathrm{R} 1$ " is presented in Fig. 3. Red and white squares in Fig. 2e denote the FOVs of Figs. 3a-d and $\mathrm{e}-1$, respectively. We find that the emission intensities of the BPs at $1330 \AA$ vary with time. At 18:41:56 UT, the intensities of the two BPs (yellow circles in Fig. 3a) were very weak. About 2 min later, the emissions of the two BPs increased by $60 \%$, and the mass upflows were simultaneously initiated (Figs. 3b, c). The constant upflows resulted in the appearance of observed loop-like structures linked with the BPs. The magnetic fields at the two BPs are very weak and could not be clearly discerned (Fig. 3d). More drastic events occasionally occurred at the concentration area of the BPs (Figs. 3e-h). At about 18:03 UT, the emission intensity of the BPs was enhanced, and meanwhile the upflow occurred with a projected velocity of about $50 \mathrm{~km} \mathrm{~s}^{-1}$. Then the intensity of the upflow was decreased at 18:08 UT. About 3 min later, the BPs and the upflow were activated again. In AIA $171 \AA$ images (panels i), j)), the outflows (e.g., see the "Flow" in panel (f)) could not be observed. Only the relatively steady EUV threads could be discerned. At the base of the upflow, magnetic flux cancellation of the strong negative polarity fields with the nearby small-scale positive polarity fields was observed (Figs. 3k, l).

The appearance of the BPs in region "R2" (Fig. 1c) was shown in Fig. 4. The loop-like structures connecting with the BPs could be observed in the 171 and $1330 \AA$ channels, corresponding to the bright EUV threads of the quiescent filament 


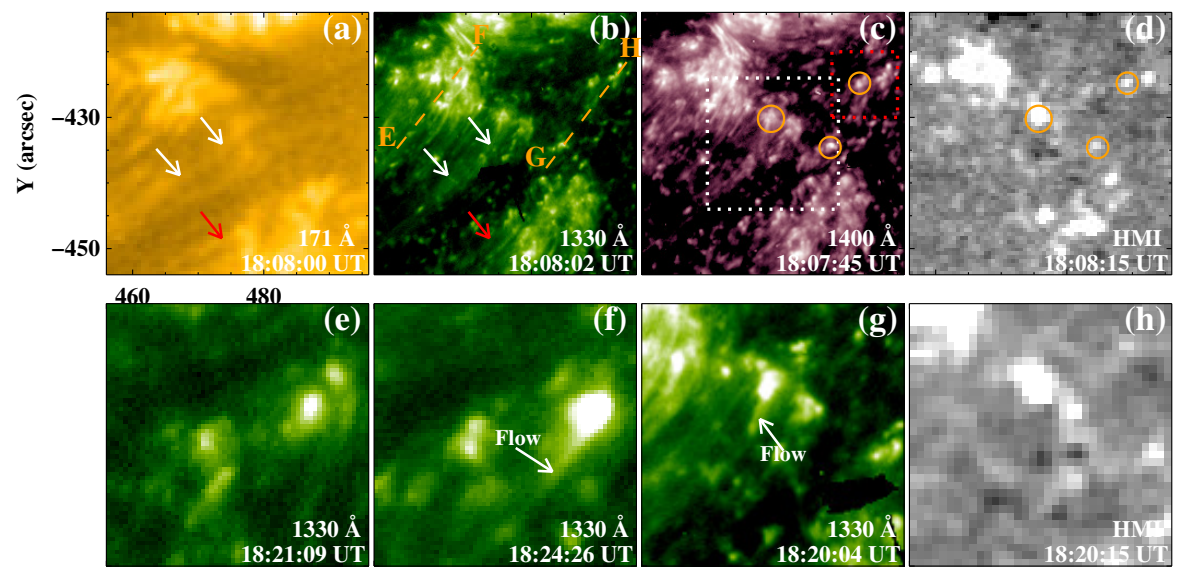

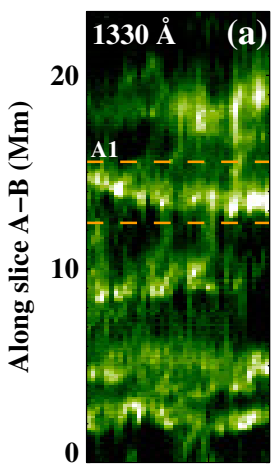

18:10 18:30

ime (

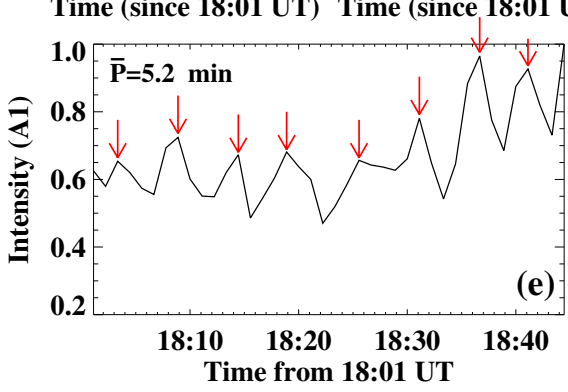

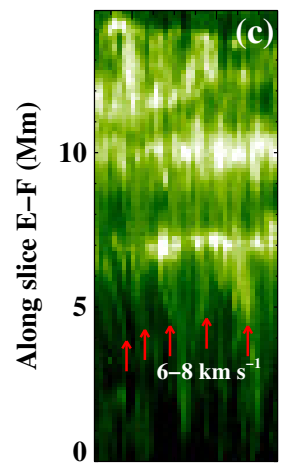

18:10 18:30

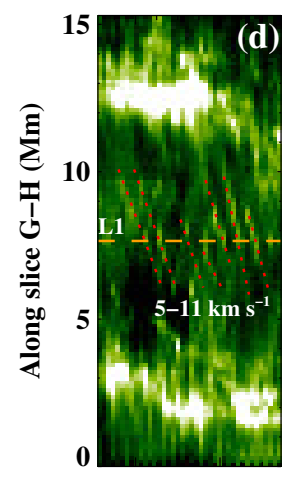

18:10 18:30
UT) Time (since 18:01 UT) Time (since 18:01 UT)

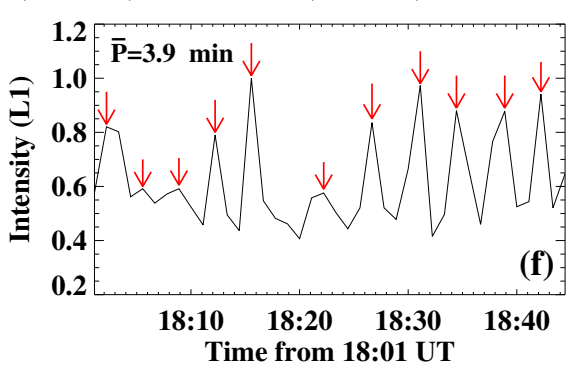

Fig. 4. Appearance of BPs and upflows in region "R2" (white square in Fig. 1c). The arrows (panels a), b)) point to the loop-like structures connecting with BPs in AIA $171 \AA$ and IRIS $1330 \AA$ images. Dashed lines " $E-F$ " and " $G-H$ " (panel b)) show the cut positions used to obtain the stack plots shown in Figs. 5c-d. The yellow circles outline the locations of several BPs in the $1400 \AA$ image and HMI LOS magnetogram. Red and white squares in panel c) denote the FOVs of panels e)-f) and $\mathbf{g})-\mathbf{h}$ ), respectively.
Fig. 5. Panels a)-d): stack plots along slices "A-B" and "C-D" (dashed lines in Fig. 2b) and slices "E-F" and "G-H" (dashed lines in Fig. 4b). Red arrows and dotted lines show the repeated upflows. Panel e): intensity variation of a BP in panel a) at $1330 \AA$. Each data point of the profile is the vertical sum of the intensity in area "A1" (two dashed lines in panel a)). Red arrows point to the peaks of the quasiperiodic intensity profile. Panel f): horizontal slice (black curve) along the dashed line ("L1") in panel d) at $1330 \AA$. (arrows in Figs. 4a, b). The direction of the loop-like structures in this region is roughly toward the southeast, which is different from the loop-like structures in region "R1" (red and white arrows in Fig. 1c). The BPs are anchored in positive polarity fields and their magnetic flux ranges from $6.9 \times 10^{17} \mathrm{Mx}$ to $1.4 \times 10^{18} \mathrm{Mx}$ (Figs. $4 \mathrm{c}, \mathrm{d}$ ). The small-scale explosive events are frequently initiated in close proximity to some BPs. After we took one BP (Figs. 4e, f) as an example, its emission intensity at $1330 \AA$ was suddenly enhanced, and meanwhile the bright material was ejected from the BP with a velocity of $10 \mathrm{~km} \mathrm{~s}^{-1}$. The $\mathrm{BP}$ and the associated upflow comprise a "tadpole-like" morphology. At 18:20:04 UT, the upflow at another BP appeared (Fig. 4g). The magnetogram showed that extremely weak negative polarity fields seemed to exist near the main positive polarity fields (Fig. 4h).

To analyze the kinematic evolution of the upflows at $1330 \AA$ in detail, we obtain the stack plots (Figs. 5a-d) along four different straight lines ("A-B" and "C-D" in Fig. 2b; "E-F" and " $\mathrm{G}-\mathrm{H}$ " in Fig. 4b). The stack plots show multiple moving intensity features, with each strip representing the propagating upflow. The velocity of the upflows initiated from the BPs was about 5-14 $\mathrm{km} \mathrm{s}^{-1}$. The emission of the BPs and the appearance of upflows are intermittent, and indeed somewhat periodic. The upflows seemed to appear each time when the emission intensity of the BP was enhanced. The vertical sum of the intensity in area "A1" (two dashed lines in Fig. 5a) was shown in Fig. 5e. The intensity of the BP exhibited a quasi-periodic pattern with an average period of about $5.2 \mathrm{~min}$. The peak intensity of the BP was about 1.4 times as high as the valley intensity. The upflows associated with the brightenings of BPs also occurred periodically. In the stack plot in region "R2" (Fig. 5d), we selected the intensity variation at location "L1" to obtain the period of the upflows. Location "L1" is far away from the north BPs, so the intensity variation of the BPs is not affecting the measured intensity of the upflows. Also, several upflows could be clearly detected at this location. It shows that the period of the upflows was about 3.9 min (black curve in Fig. 5f).

Figure 6 shows the profiles of Si IV $1402.77 \AA$ line at locations of the upflows from the BPs. We are interested mostly in the main component of the line, including the line shift and the line width. Therefore we add a parabolic background in the single Gaussian fit just to account for the wing emission. The FWHW 

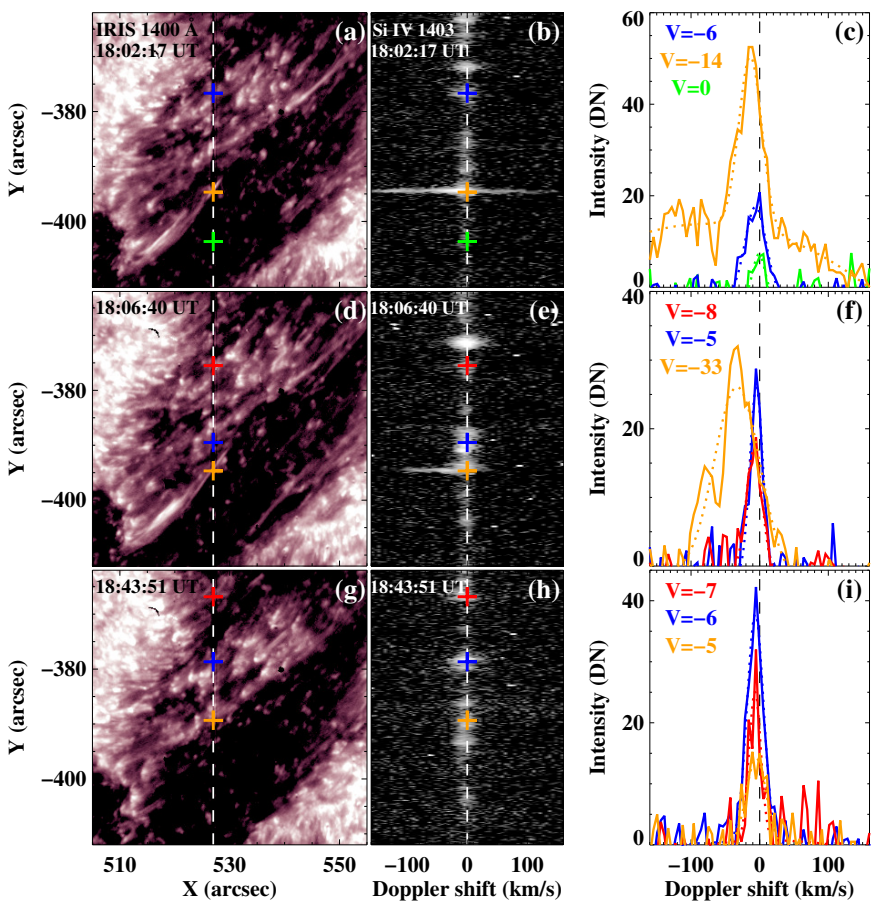

Fig. 6. IRIS $1400 \AA$ A images (left column), Si IV $1402.77 \AA$ Å spectra (middle column), and profiles of the Si IV line (right column) at the selected locations. The FOV of $1400 \AA$ images is the same as that of Fig. 2. The red, blue, and yellow pluses denote the locations of the upflows initiated from the BPs. The green plus in panel a) indicates one location without upflows. Dotted curves in right column are the single Gaussian fitting with a parabolic background of the solid curves.

of the fitting profile is thought to be the line width (Peter et al. 2014). The recurrent upflows generated from the same BP have different Doppler shifts and line widths. At 18:02:17 UT, the emission profile at the notable upflow (yellow plus in Fig. 6a) was blueshifted by $\sim 14 \mathrm{~km} \mathrm{~s}^{-1}$ with the Doppler width of about $40 \mathrm{~km} \mathrm{~s}^{-1}$ (Figs. 6b, c). For using a single Gaussian function with a parabolic background, the fitting profile at this location (yellow dotted line in panel c) looks asymmetric and shows a blue-wing excess. One could also have used a Gaussian function for the extended wings (Peter 2000), but in the present case the parabolic fit works as well. Inclusion of a second broad component could also have an impact on the shift in the main component. The broad component that we account for by the parabolic background could be that some other high-velocity component is present (indicating jets or whatever; see Peter 2001, 2010), but this contributes less to the emission than the main component of the spectrum. The emission intensity of the second upflow at the same location was decreased by $40 \%$ at 18:06:40 UT (yellow pluses and curves in Figs. 6d-f). However, the blueshift of the second upflow reached about $33 \mathrm{~km} \mathrm{~s}^{-1}$ and the line width was increased by a factor of two. Afterwards, the upflows exhibited low blueshifts of 5-7 $\mathrm{km} \mathrm{s}^{-1}$ and had the line width of about 20-30 $\mathrm{km} \mathrm{s}^{-1}$ (Figs. 6g-i).

\section{Summary and discussion}

We first report the observations of subarcsecond BPs underlying a quiescent filament in the TR by the IRIS on 2015 February 13. The BPs have a spatial scale of about $350-580 \mathrm{~km}$ and a lifetime of more than several tens of minutes. The dimension of BPs is similar to those of the EUV bright dots at the edge of the active region (Régnier et al. 2014) and the subarcsecond bright dots in the TR above sunspots (Tian et al. 2014a), however, the lifetime of BPs is much longer than those of the reported bright dots ( 25 s). Compared to well-known CBPs, the BPs in the filament channel are much smaller than CBPs (with a spatial size of 5-20"; Vaiana et al. 1973). The subarcsecond BPs and CBPs are both long-lived, and their emission intensities both exhibit the quasi-periodic variation. In this work, the intensities of BPs have a period of about 4-5 min, comparable to the minimum period of CBPs. The BPs are anchored at small-scale magnetic structures near the PIL, and their magnetic flux was about $10^{17}-10^{18} \mathrm{Mx}$, almost two orders of magnitude smaller than that of CBPs.

The directions of upflows are almost parallel to the spine of the quiescent filament. The upflows initiated from the BPs with opposite polarity magnetic fields have opposite directions (Fig. 1c). The direction of the upflows is generally thought to trace out the connectivity of the magnetic fields. The bidirectional upflows probably imply the consistent direction of magnetic fields along the filament spine. The filament is composed of multiple groups of magnetic systems and their directions are the same, pointing from the northwest to the southeast (Fig. 1). The connectivity between magnetic structures within the filament channel is not in any arbitrary pattern, e.g., the positive magnetic structures in "R2" connect with the southeast negative ones and the negative magnetic structures in "R1" connect with the northwest positive ones (Figs. 1c and e). Martin (1998) found that the chromospheric fibrils on the two sides of an $\mathrm{H} \alpha$ filament had opposite orientations. Sheeley et al. (2013) report on cellular plumes leaning in opposite directions on the two sides of a filament channel based on SDO observations. The configuration of the upflows within the filament channel in our work is roughly consistent with those shown in previous studies and may be an intrinsic feature of the quiescent filament. The multiple groups of magnetic systems along the filament spine have the same orientation, and this magnetic topology is more stable than the opposite field orientations within the filament due to the low probability of magnetic reconnection.

The BPs appear to be the bases of filament threads, and the upflows intermittently inject plasma inside the fine threads. Our observations imply that the source of the filament material may be the recurrent upflows in the TR. Chae (2003) and Liu et al. (2005) reported the findings of $\mathrm{H} \alpha$ jets associated with the filaments and suggest that the frequent injection of flow from below supplies the mass necessary for the formation of the filaments. In our work, the quasi-periodic upflows might be able to convey mass into the filament threads, which is consistent with the injection models that the filament plasma is lifted from the lower atmosphere by magnetic reconnection (Wang 1999; Litvinenko et al. 2007).

Quasi-periodic upflows are generated when the intensities of BPs are enhanced, with the BPs and jet-like upflows composing a morphology of "tadpoles". The quasi-periodic brightenings of BPs and appearances of upflows might be caused by oscillatory magnetic reconnection (Kankelborg et al. 1997; Gupta \& Tripathi 2015). The recurring time scale of the upflows is about 4-5 min, which is approximately consistent with the period of solar p-mode waves. This implies that p-mode waves possibly modulate magnetic reconnections by changing the plasma density near the reconnection site and induce periodic reconnection of the magnetic fields (Chen \& Priest 2006). The brightenings of BPs and generation of upflows are observed each time when the magnetic reconnection occurs. Recently, Li \& Zhang (2015) found quasi-periodic oscillations of 
the small-scale substructures within the flare ribbon with periods of 3-6 min, which is attributed to quasi-periodic slipping reconnection. The observation of wave-like pulses in a filament pillar was presented and simulated by Schmieder et al. (2013) and Ofman et al. (2015). These waves have a period of about $300 \mathrm{~s}$, similar to those of quasi-periodic upflows in our event. They interpret these waves as propagating perpendicular to the filament magnetic field as nonlinear fast magnetosonic waves in the filament foot. In this work, the velocity of the upflows in the plane of sky is about $5-50 \mathrm{~km} \mathrm{~s}^{-1}$. The emission line of Si IV $1402.77 \AA$ at the locations of upflows exhibits obvious blueshifts of about $5-30 \mathrm{~km} \mathrm{~s}^{-1}$, and the line profile is broadened with the width of more than $20 \mathrm{~km} \mathrm{~s}^{-1}$. The Doppler velocities of the repeated upflows at the same location are not constant, and they change with time. This may be caused by the different energy released during the magnetic reconnection process. The more energy released, the higher velocity of the upflow.

High-resolution observations showed that filament threads had an average width of 0"3 (Lin et al. 2005; Li \& Zhang 2013), which is slightly thinner than the spatial scale of subarcsecond BPs $\left(00^{\prime} 48-00^{\prime \prime} 8\right)$. The plasma in the filament threads moves with the magnetic field lines in opposite directions, appearing as "counter-streaming" flows (Zirker et al. 1998; Schmieder et al. 2010; Alexander et al. 2013). The speed of the "counterstreaming" flows is about 5-20 km s${ }^{-1}$, comparable to that of quasi-periodic upflows at the bases of filament threads. Until now, the mass source and triggering mechanism of anti-parallel flows are still unknown. Chen et al. (2014) suggest that the $\mathrm{H} \alpha$ counter-streamings in previous observations were caused by longitudinal oscillations of filament threads. Our observations reveal that the upflows at the BPs with opposite polarity magnetic fields move along the filament threads in opposite directions. The bidirectional upflows travelling from the positive and negative-polarity footpoints of filament threads indicate that the filament is composed of multiple separate systems of magnetic fields. The "counter-streaming" flows may be caused by the propagation of bidirectional upflows along different magnetic systems that are initiated at the subarcsecond BPs below the filament.

Acknowledgements. IRIS is a NASA small explorer mission developed and operated by LMSAL with mission operations executed at NASA Ames Research center and major contributions to downlink communications funded by the Norwegian Space Center (NSC, Norway) through an ESA PRODEX contract. We acknowledge the SDO/AIA and HMI for providing data. This work is supported by the National Natural Science Foundations of China (11303050, 11533008 and 1221063) and the Strategic Priority Research Program-The Emergence of Cosmological Structures of the Chinese Academy of Sciences, Grant No. XDB09000000.

\section{References}

Alexander, C. E., Walsh, R. W., Régnier, S., et al. 2013, ApJ, 775, L32 Berger, T. E., Liu, W., \& Low, B. C. 2012, ApJ, 758, L37 Chae, J. 2003, ApJ, 584, 1084

Chae, J., Martin, S. F., Yun, H. S., et al. 2001, ApJ, 548, 497

Chen, P. F., \& Priest, E. R. 2006, Sol. Phys., 238, 313

Chen, P. F., Harra, L. K., \& Fang, C. 2014, ApJ, 784, 50

De Pontieu, B., Title, A. M., Lemen, J. R., et al. 2014, Sol. Phys., 289, 2733

Doyle, J. G., Popescu, M. D., \& Taroyan, Y. 2006, A\&A, 446, 327

Golub, L., Krieger, A. S., Silk, J. K., Timothy, A. F., \& Vaiana, G. S. 1974, ApJ, 189, L93

Golub, L., Krieger, A. S., \& Vaiana, G. S. 1976, Sol. Phys., 50, 311

Gupta, G. R., \& Tripathi, D. 2015, ApJ, 809, 82

Habbal, S. R., Withbroe, G. L., \& Dowdy, J. F., Jr. 1990, ApJ, 352, 333

Heinzel, P., Schmieder, B., \& Tziotziou, K. 2001, ApJ, 561, L223

Hong, J., Jiang, Y., Yang, J., et al. 2014, ApJ, 796, 73

Kankelborg, C. C., Walker, A. B. C., II, \& Hoover, R. B. 1997, ApJ, 491, 952

Karpen, J. T., Tanner, S. E. M., Antiochos, S. K., \& DeVore, C. R. 2005, ApJ, 635, 1319

Lemen, J. R., Title, A. M., Akin, D. J., et al. 2012, Sol. Phys., 275, 17

Li, T., \& Zhang, J. 2013, ApJ, 770, L25

Li, T., \& Zhang, J. 2015, ApJ, 804, L8

Li, D., Ning, Z. J., \& Wang, J. F. 2013, New Astron., 23, 19

Li, L. P., Peter, H., Chen, F., \& Zhang, J. 2014, A\&A, 570, A93

Li, T., Zhang, J., \& Ji, H. 2015, Sol. Phys., 290, 1687

Lin, Y., Engvold, O., Rouppe van der Voort, L., Wiik, J. E., \& Berger, T. E. 2005, Sol. Phys., 226, 239

Litvinenko, Y. E., Chae, J., \& Park, S.-Y. 2007, ApJ, 662, 1302

Liu, Y., Kurokawa, H., \& Shibata, K. 2005, ApJ, 631, L93

Liu, W., Berger, T. E., \& Low, B. C. 2012, ApJ, 745, L21

Madjarska, M. S., Doyle, J. G., Teriaca, L., \& Banerjee, D. 2003, A\&A, 398, 775

Martin, S. F. 1998, Sol. Phys., 182, 107

Ning, Z., \& Guo, Y. 2014, ApJ, 794, 79

Ofman, L., Knizhnik, K., Kucera, T., \& Schmieder, B. 2015, ApJ, 813, 124

Parenti, S. 2014, Liv. Rev. Sol. Phys., 11, 1

Pesnell, W. D., Thompson, B. J., \& Chamberlin, P. C. 2012, Sol. Phys., 275, 3

Peter, H. 2000, A\&A, 360, 761

Peter, H. 2001, A\&A, 374, 1108

Peter, H. 2010, A\&A, 521, A51

Peter, H., Tian, H., Curdt, W., et al. 2014, Science, 346, 1255726

Pikel'Ner, S. B. 1971, Sol. Phys., 17, 44

Priest, E. R., van Ballegooijen, A. A., \& Mackay, D. H. 1996, ApJ, 460, 530

Régnier, S., Alexander, C. E., Walsh, R. W., et al. 2014, ApJ, 784, 134

Saito, K., \& Tandberg-Hanssen, E. 1973, Sol. Phys., 31, 105

Samanta, T., Banerjee, D., \& Tian, H. 2015, ApJ, 806, 172

Schmieder, B., Chandra, R., Berlicki, A., \& Mein, P. 2010, A\&A, 514, A68

Schmieder, B., Kucera, T. A., Knizhnik, K., et al. 2013, ApJ, 777, 108

Scherrer, P. H., Schou, J., Bush, R. I., et al. 2012, Sol. Phys., 275, 207

Sheeley, N. R., Jr., Martin, S. F., Panasenco, O., \& Warren, H. P. 2013, ApJ, 772, 88

Tian, H., Kleint, L., Peter, H., et al. 2014a, ApJ, 790, L29

Tian, H., DeLuca, E., Reeves, K. K., et al. 2014b, ApJ, 786, 137

Vaiana, G. S., Davis, J. M., Giacconi, R., et al. 1973, ApJ, 185, L47

van Ballegooijen, A. A., \& Martens, P. C. H. 1989, ApJ, 343, 971

Wang, H., Chae, J., Gurman, J. B., \& Kucera, T. A. 1998, Sol. Phys., 183, 91

Wang, Y.-M. 1999, ApJ, 520, L71

Xia, C., Chen, P. F., \& Keppens, R. 2012, ApJ, 748, L26

Zhang, Q. M., Chen, P. F., Guo, Y., Fang, C., \& Ding, M. D. 2012, ApJ, 746, 19

Zirker, J. B., Engvold, O., \& Martin, S. F. 1998, Nature, 396, 440 